\title{
Palliation of proximal malignant biliary obstruction by endoscopic endoprosthesis insertion
}

\author{
A A Polydorou, S R Cairns, J F Dowsett, A R W Hatfield, P R Salmon, P B Cotton, \\ R C G Russell
}

\section{Abstract}

For four years up to December 1987, 190 patients (median age 73 years) with proximal malignant biliary obstruction were treated by endoscopic endoprosthesis insertion. Altogether 101 had cholangiocarcinoma, 21 gall bladder carcinoma, 20 local spread of pancreatic carcinoma, and 48 metastatic malignancy. Fifty eight patients had type I, 54 type II, and 78 type III proximal biliary strictures (Bismuth classification). All patients were either unfit or unsuitable for an attempt at curative surgical resection. A single endoprosthesis was placed initially, with a further stent being placed only if relief of cholestasis was insufficient or sepsis developed in undrained segments. The combined percutaneous-endoscopic technique was used to place the endoprosthesis when appropriate, after failed endoscopic endoprosthesis insertion or for second endoprosthesis placement. Full follow up was available in $97 \%$. Thirteen patients were still alive at the time of review and all but one had been treated within the past six months. Initial endoprosthesis insertion succeeded technically at the first attempt in 127 patients, at the second in 30 , and at a combined procedure in a further 13 (cumulative total success rate $89 \%$ - type I: $93 \%$; type II: $94 \%$; and type III: $84 \%$ ). There was adequate biliary drainage after single endoprosthesis insertion in 152 of the 170 successful placements, giving an overall successful drainage rate of $80 \%$. Three patients had a second stent placed by combined procedure because of insufficient drainage, giving an overall successful drainage rate of $82 \%$ ( 155 of 190 ). The final overall drainage success rates were type I: $91 \%$; type II: $83 \%$; and type III: $73 \%$. The early complication rates were type I: $7 \%$; type II: $14 \%$; and type III: $31 \%$. The principal early complication was clinical cholangitis, which occurred in 13 patients $(7 \%)$ and required second stent placement in five. The $\mathbf{3 0}$ day mortality was $22 \%$ overall (type I: $14 \%$; type II: $15 \%$; and type III: $32 \%$ ) but the direct procedure related mortality was only 3\%. Median survival overall for types I, II, and III strictures were 21,12 , and 10 weeks respectively but survival was significantly shorter for metastatic than primary malignancy $(p<0.05)$. Endoscopic insertion of a single endoprosthesis will provide good palliation of proximal malignant biliary obstruction caused by unresectable malignancy in $80 \%$ of patients. Second stents should be placed only if required. Extensive stricturing because of metastatic disease carries a poor prognosis and careful patient selection for treatment is required.
Although management of distal malignant biliary obstruction by endoprosthesis insertion is well established, ' the place of endoscopic endoprosthesis insertion for proximal malignant biliary obstruction (Fig 1) remains controversial. Surgery, involving hepatic resection if necessary, offers the only chance of long term cure but carries considerable procedure related morbidity and mortality. ${ }^{2}$ Most $(90 \%)$ patients are, however, unsuitable or unfit for attempted curative resection. ${ }^{3}$ Palliative options include surgical bypass and surgical percutaneous or endoscopic endoprosthesis insertion. ${ }^{3-7}$ Internal drainage should always be preferred to external drainage. ${ }^{89}$ Drainage may, if desired, be supplemented by radiotherapy. ${ }^{1011}$

Attempts at palliation, even in elderly patients, are usually worthwhile because the quality of life may be improved considerably by the relief of the pruritus and nausea of cholestasis. As surgical bypass procedures, in the best hands and in the fittest patients, carry a $33 \%$ operative mortality ${ }^{2}$ and surgical intubation carries a 60 day postoperative morbidity of $26 \%$ and a $30 \%$ mortality, $^{3}$ a strong argument can be made for management by percutaneous or endoscopic endoprosthesis insertion. Large studies of both techniques in single centres have been few. A randomised trial from this institution, however, suggested a lower complication rate with endoscopic than percutaneous insertion, and the endoscopic route is therefore the preferred primary intervention. ${ }^{12}$ Moreover, the development of the combined percutaneous-endoscopic technique $(C P)$ has provided an increased endoscopic
Type I
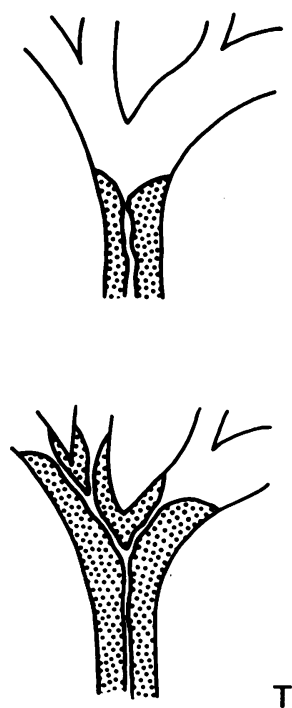

Type III 
success rate, while still avoiding large bore hepatic puncture. ${ }^{13}$ It has become our second line intervention.

We report our experience with endoscopic endoprosthesis placement in 190 patients with either primary or secondary proximal malignant biliary obstruction at the hilum. In the analysis we have attempted to address several points of importance to the clinician. These include the initial success rate, the number of endoprostheses required to provide adequate relief of cholestasis, the relative merits of stent placement into the left or right lobe, the prevalence of early and late septic complications, the prevalence of late stent change either because of stent blockage or tumour progression with overgrowth, and finally the value of stenting patients with secondary malignancy.

\section{Methods}

Between October 1983 and December 1987, 190 consecutive patients with biliary obstruction at the hilum because of primary or secondary malignancy were treated by attempted endoscopic endoprosthesis insertion. Details, including intention to treat and the result of treatment, were prospectively entered onto a computer programme (PEDRO). There were 74 men and 116 women with a median (range) age of 73 (3594 ) years. The mean (range) duration of jaundice before the first endoscopy was 5 (1-14) weeks and the mean (range) serum bilirubin was 361 (22913) $\mathrm{mmol} / \mathrm{l}$, normal $<17$. Other data included a mean (range) serum alkaline phosphatase of 1540 (360-4600) IU/l, normal $<280$, mean (range) serum albumin of $34(20-43) \mathrm{g} / 1$, normal $35-53$, mean (range) serum creatinine of $106(82-420)$ $\mu \mathrm{mol} / \mathrm{l}$, normal $50-125$, and mean (range) haemoglobin of $11 \cdot 7(8 \cdot 0-14 \cdot 3) \mathrm{g} / \mathrm{dl}$.

All patients were considered unsuitable for resection on the basis of medical fitness or tumour extent, or both, as defined by ultrasound, computed tomogram (tumour extending into both lobes or major vascular involvement) or cholangiography (type II/III obstruction), or both. The proximal obstruction was subdivided according to Bismuth's classification on the basis of cholangiographic or ultrasound information (Fig 1), or both, and according to proved or presumed histology (Table I). It is stressed that no attempt was made at any stage to obtain a 'complete' diagnostic cholangiogram. Contrast medium injection before stricture passage was stopped once a duct deemed suitable for stenting was identified and was continued only after

TABLE I Type of stricture in study group

\begin{tabular}{lllll}
\hline \multicolumn{5}{c}{ Type of biliary obstruction } \\
\cline { 2 - 5 } & $I$ & $I I$ & $I I I$ & Total \\
\hline No (\%) of patients & $58(31)$ & $54(28)$ & $78(41)$ & 190 \\
$(M / F)$ & $19 / 39$ & $23 / 31$ & $32 / 46$ & $74 / 16$ \\
Mean age (years) (range) & $72(41-94)$ & $70(35-91)$ & $71(42-92)$ & $71(35-94)$ \\
Mean bilirubin $(\mu \mathrm{mol} / \mathrm{l})$ & $292(22-714)$ & $444(140-913)$ & $343(31-728)$ & $361(22-913)$ \\
(range) & 25 & 28 & 48 & 101 \\
Cholangiocarcinoma & 12 & 5 & 3 & 20 \\
Pancreatic carcinoma & 14 & 5 & 18 & 48 \\
Metastatic carcinoma & 7 & 5 & 9 & 21 \\
Gall bladder carcinoma & & & & \\
\hline
\end{tabular}

${ }^{\star}$ Bismuth classification. secure access to the desired segment was obtained with a 5 FG catheter. The rationale behind this approach was to avoid contrast medium injection into segments that would not be adequately drained by the inserted stent. One stent only was placed in all but two patients, in whom the first segment stented was thought inadequate at the time of initial stenting.

All patients received parenteral antibiotics (usually mezlocillin $2 \mathrm{~g}$, eight hourly, given one hour before the procedure and continued for at least 36 hours after successful stent placement or until successful drainage was achieved). An experienced senior endoscopist was present during most of the procedures. Amsterdam type 10 FG polyethylene endoprostheses were routinely inserted but $10 \%$ of patients had 8 or $11.5 \mathrm{FG}$ stents placed either initially or at stent change. Stents were inserted over a guide wire and coaxial catheter via an Olympus TJF10 $4 \cdot 2$ $\mathrm{mm}$ channel endoscope, using standard techniques. ${ }^{14}$ Endoscopic sphincterotomy was performed in most patients both to assist stent insertion and to facilitate later change. When a stent could not be placed endoscopically, the combined percutaneous-endoscopic technique (CP) was used. ${ }^{15}$ All patients were carefully monitored clinically after the procedures with particular attention to fever, serial biochemistry, and ultrasound evidence of decompression. Patients were withdrawn from repeated attempts at palliation and designated 'failed palliation' if non-specific general deterioration occurred or if, on discussion with the patient, the risks of further intervention were agreed to outweigh the possible benefit.

Successful endoprosthesis placement was defined as passage of the prosthesis across the stricture with good radiological positioning, immediate bile passage down the stent, and aerocholia on subsequent plain $x$ ray or ultrasound. ${ }^{1617}$ Most patients also had segment decompression assessed by ultrasonography, usually 36-72 hours after the procedure.

Successful drainage was defined as loss of pruritus, if present, plus a fall in the bilirubin concentration greater than $30 \%$ of the pretreatment value within 30 days (but usually within 10 days). It should be stressed that the aim was reduction in bilirubin to acceptable concentrations (usually $<100 \mu \mathrm{mol} / \mathrm{l}$ ) where it was likely to relieve symptoms of cholestasis such as pruritus and nausea. ${ }^{18}$

Early complications were defined as those occurring within 30 days of endoprosthesis placement. Cholangitis was diagnosed if a fever above $38^{\circ} \mathrm{C}$ developed without other cause and was associated with rigors or persisted for longer than 48 hours after the procedure. That is, postprocedural fever on the night of the procedure, which is undoubtedly caused by a brief bacteraemia and settles rapidly either spontaneously or because of antibiotic prophylaxis, was not labelled cholangitis. Complications of percutaneous biopsies are not included and did not contribute to the overall mortality and morbidity.

Thirty day mortality was defined as death within 30 days of the first attempt at endoscopic endoprosthesis insertion, whether successful or 
TABLE II Results in 190 patients with proximal biliary obstruction treated by endoscopic endoprosthesis insertion

\begin{tabular}{|c|c|c|c|c|}
\hline & \multicolumn{4}{|c|}{ Type of stricture ${ }^{\star}$} \\
\hline & $I$ & $I I$ & III & Total \\
\hline \multicolumn{5}{|l|}{ No of patients } \\
\hline lst attempt & 58 & 54 & 78 & 190 \\
\hline \multicolumn{5}{|l|}{ Technical success: } \\
\hline lst attempt (S/F) & $45 / 13(78 \%)$ & $36 / 18(67 \%)$ & $46 / 32(59 \%)$ & $127 / 63(67 \%)$ \\
\hline No further attempt & & & & 16 \\
\hline 2nd attempt (S/F) & $5 / 4(9 \%)$ & $12 / 1(22 \%)$ & $13 / 5(17 \%)$ & $30 / 10(16 \%)$ \\
\hline Combined procedure $(S / F)$ & $4 / 0(7 \%)$ & $3 / 1(6 \%)$ & $6 / 2(8 \%)$ & $13 / 3(7 \%)$ \\
\hline Total & $54(93 \%)$ & $51(94 \%)$ & $65(84 \%)$ & $170(89 \%)$ \\
\hline \multicolumn{5}{|l|}{ Successful drainage: } \\
\hline Single stent & $53(91 \%)$ & $44(81 \%)$ & $55(71 \%)$ & $152(80 \%)$ \\
\hline Two stents & & $1(2 \%)$ & $2(3 \%)$ & $155(82 \%)$ \\
\hline \multicolumn{5}{|l|}{ Early complications: } \\
\hline No of patients & $4(7 \%)$ & $8(15 \%)$ & $24(31 \%)$ & $36(19 \%)$ \\
\hline No of events & $6(10 \%)$ & $16(30 \%)$ & $28(36 \%)$ & $50(26 \%)$ \\
\hline 30 Day mortality & $8(14 \%)$ & $8(15 \%)$ & $25(32 \%)$ & $41(22 \%)$ \\
\hline \multicolumn{5}{|l|}{ Survival: } \\
\hline Mean (wks) & & & & \\
\hline Median (range) (wks) & $21(2-85)$ & $12(1-94)$ & $10(1-115)$ & $12(1-115)$ \\
\hline \multicolumn{5}{|l|}{ Stent change: } \\
\hline No of patients & $27(47 \%)$ & $25(46 \%)$ & $28(36 \%)$ & $80(42 \%)$ \\
\hline No of events $(\mathrm{S} / \mathrm{F})$ & $48 / 4$ & $36 / 5$ & $46 / 11$ & $130 / 20$ \\
\hline
\end{tabular}

$\star$ Bismuth classification. in Table II, 16 patients had no further attempts after a failed first procedure (type I: four; type II: two; type III: 10). Seven patients proceeded to $\mathrm{CP}$ immediately after an initial failure of endoscopic stent placement (type II: three; type III: four), and all patients except one who had a type III obstruction proceeded to CP after a second failed endoscopic attempt. The overall success rate for stent placement was 170 of $190(89 \%)$ (Table II). Of the 20 patients with failed stent placement, two had external drainage only after failed CP and the remaining 18 patients had no further intervention. The site of stent placement was identified in 116 patients with type II or III strictures. In 70 patients the stent was placed in the right hepatic duct and in 39 the left duct. No definite location was identified in the remaining seven cases.

Successful drainage was obtained in $80 \%$ of patients overall (type I: $91 \%$; type II: $81 \%$; type III: $71 \%$ ) (Table II). That is, inadequate relief of cholestasis despite adequate placement of a single stent occurred overall in $7 \%$ of patients $(2 \%$, $13 \%$, and $13 \%$ for types I, II, and III strictures respectively). Failure of drainage was significantly more common after technically successful endoprosthesis placement in type III than type I obstruction $(\mathrm{p}<0.01)$. A second stent was placed by CP in three patients (type II: one; type III: two) because of failure of resolution of jaundice despite segmental decompression by the first stent - all of which provided successful drainage. The success rate of drainage overall thus increased to $83 \%$ for type II strictures and $74 \%$ for type III strictures. attempted only after biliary decompressio was obtained in 108 patients $(57 \%)$ either by aspiration cytology or by Biopty gun trucut biopsy. This was normally done at the time of the ultrasonographic decompression check. Diagnosis of the histological origin of the biliary obstruction was surmised from available clinical and imaging data in the remainder of cases.

Follow up was obtained by examination of the case notes and from referring consultants and general practitioners. Statistical analysis was performed using $\chi^{2}$ with Yates's correction and Mann-Whitney U test.

\section{Results}

ENDOPROSTHESIS PLACEMENT AND FUNCTION Endoscopic placement of an endoprosthesis was achieved in 127 patients at the first attempt, in 30 at the second, and in 13 patients at CP. As shown

TABLE III Early complications in 190 patients with proximal malignant biliary obstruction treated by endoscopic endoprosthesis insertion

\begin{tabular}{lllll}
\hline \multirow{2}{*}{ Complication } & \multicolumn{4}{l}{ Type of biliary obstruction ${ }^{\star}$} \\
\cline { 2 - 5 } & $I$ & $I I$ & $I I I$ & Total \\
\hline Cholangitis & 2 & 3 & 8 & 13 \\
Pancreatitis & - & - & 3 & 3 \\
Retroperitoneal & & 2 & 3 & 7 \\
perforation & 2 & 2 & 5 & 7 \\
Gastrointestinal & & 2 & 5 & 8 \\
bleeding & - & 4 & 2 & 8 \\
Stent migration & 2 & 3 & 5 & 2 \\
Bile leakage & - & 1 & 1 & 2 \\
Respiratory failure & - & 1 & 1 & \\
Renal failure & - & 1 & & \\
\hline
\end{tabular}

$\star$ Bismuth classification

\section{EARLY COMPLICATIONS}

Complications occurring within 30 days of the procedure were present in 36 patients (19\%) (Table III). Cholangitis was the principal major complication occurring in $13(7 \%)$, and if not associated with endoprosthesis insertion failure, it was presumed to be caused by sepsis in undrained segments. Cholangitis resolved on parenteral antibiotics alone in five patients, with replacement of the single stent in three, and in five the placement of an additional second stent into the presumed undrained segment was performed (this was achieved endoscopically in two and via $C P$ in three patients). All $C P$ were performed via the ducts of the left lobe of the liver and all three patients had had initial endoducts.

The prevalence of complications after stent placement in patients with type II or III obstruction was not significantly different in patients with an endoprosthesis placed in the right ducts compared with those with an endoprosthesis placed in the left hepatic duct $(p>0.05)$.

\section{MORTALITY}

Follow up to 30 days was obtained for all patients. The 30 day mortality was $22 \%$ overall (41 patients) and was $14 \%$ for type I obstruction, $15 \%$ for type II, and $32 \%$ for type III (Table II). There was a significant difference in 30 day mortality between patients presenting with type I scopic endoprosthesis insertion into right lobe 

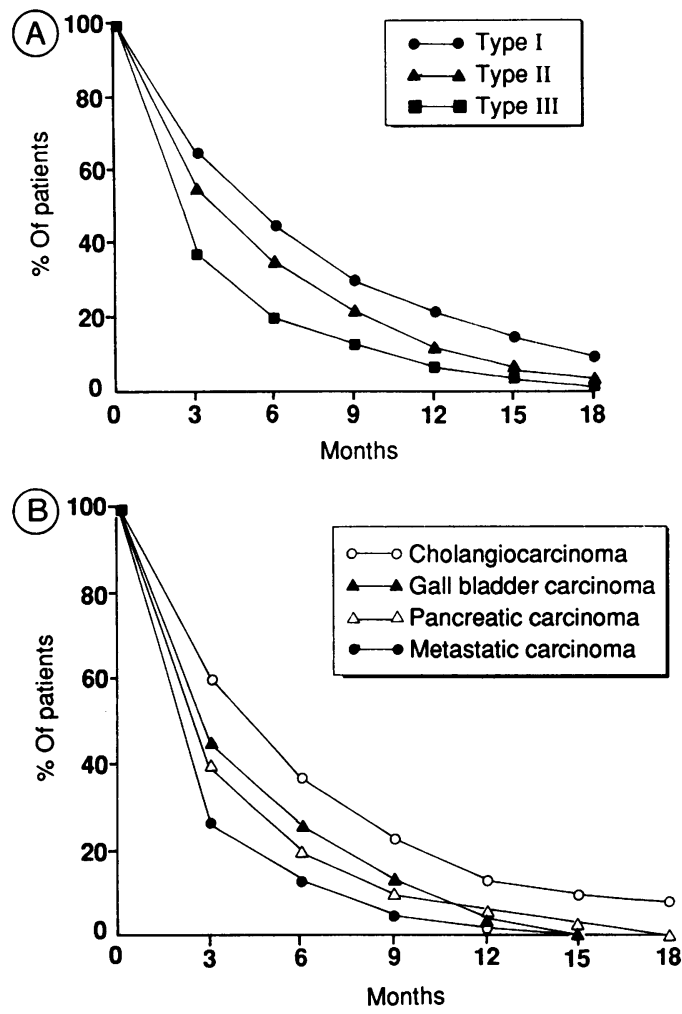

Figure 2: (A) Patient survival according to type of stricture. Actuarial 18 month survival after endoscopic placement of endoprosthesis in patients with unresectable malignant liver hilar strictures $(n=172) . P(50 \%$ survival $): I$ v $I I=0 \cdot 288 ; I$ v $I I I=0 \cdot 006 ; I I$ v $I I I=0 \cdot 162$. (B) Patient survival according to cause of stricture. Actuarial survival of patients with hilar stricture who were dead at the time of review, according to histological diagnosis $(n=172)$.

and type III hilar stricturing $(\mathrm{p}<0.05)$ and between patients with type II and type III strictures $(p<0.05)($ Fig 2$)$.

Death within 30 days was caused by a procedure related complication in five patients (3\%) (bleeding four, sepsis one). The remaining patients died from progressive malignant disease rather than a complication of the procedure.

\section{SURVIVAL}

Thirteen patients were alive at the time of review. All except one had been treated within the previous six months. The single long term survivor still alive was first treated 132 weeks before this review. Five patients were lost to long term follow up. Data about patients who have died (172) is provided in Table II. All patients who did not have biliary drainage established died within six weeks. Survival was significantly longer for type I than type III obstruction ( $<<0.01)$ (Fig 2$)$. Shorter survival was seen in patients with obstruction caused by metastatic disease or pancreatic carcinoma than in those with either cholangiocarcinoma or gall bladder carcinoma

TABLE IV Median and mean survival and 30 day mortality according to extent and cause of hilar stricture. (Only patients with a histological diagnosis are included, $n=108$.)

\begin{tabular}{|c|c|c|c|c|c|}
\hline & \multicolumn{2}{|c|}{ Mean (median) (weeks) } & \multirow[t]{2}{*}{$p$} & \multicolumn{2}{|c|}{30 day mortality $(\%)$} \\
\hline & $C+G B$ & $P+M$ & & $C+G B$ & $P+M$ \\
\hline $\begin{array}{l}\text { I } \\
\text { II } \\
\text { III }\end{array}$ & $\begin{array}{l}26(22) \\
23(16) \\
19(12)\end{array}$ & $\begin{array}{c}19(12) \\
17(10) \\
8(6)\end{array}$ & $\begin{array}{l}(0.291) \\
(0.507) \\
(0.048)\end{array}$ & $\begin{array}{l}10 \\
12 \\
28\end{array}$ & $\begin{array}{l}19 \\
23 \\
42\end{array}$ \\
\hline
\end{tabular}

$\mathrm{C}=$ cholangiocarcinoma $; \mathrm{GB}=$ gall bladder cancer $\mathrm{P}=$ pancreatic cancer $\mathbf{M}=$ metastatic cancer .
(Table IV; Fig 2). Survival of patients with successfully drained type II or III obstruction was not significantly longer in those treated by insertion of a stent in the left rather than the right hepatic duct system $(\mathrm{p}>0.05)$.

\section{LATE COMPLICATIONS}

Eighty patients required between one and six stent changes (one change: 54 patients; two changes: 13 patients; >two changes: 13 patients) (Table II).

\section{Discussion}

There are numerous alternative palliative treatments available for patients with proximal malignant biliary obstruction for whom attempted curative surgical resection is not possible..$^{192}$ These include surgical bypass ${ }^{2421}$ and surgical, ${ }^{322}$ percutaneous, ${ }^{512}$ and endoscopic ${ }^{67172023}$ endoprosthesis insertion. Internal drainage is preferred to external drainage for fluid and electrolyte, tumour seeding, and cosmetic/psychological reasons, though it probably carries a higher risk of sepsis. ${ }^{24}$ Extensive obstructive stricturing is not, however, amenable to conventional surgical bypass and requires special surgical techniques. ${ }^{25}$ Although surgical bypass offers the possibility of a single permanent definitive palliative procedure, it carries a high procedural morbidity and mortality, ${ }^{221}$ has only been reported in the minority subgroup of fit, younger patients, and although generally technically straightforward, it involves a general anaesthetic and tissue dissection. Surgical intubation has been performed when surgical bypass has not been possible and carries all the disadvantages of surgery and no proved benefit over the non-surgical alternatives. ${ }^{3}$ For most patients, who are either elderly, unfit, or have extensive bilobar disease, percutaneous or endoscopic endoprosthesis insertion are valid and often the only alternatives. As a randomised study has shown the complication rate to be lower with the latter, ${ }^{12}$ endoscopic stent placement is now our treatment of choice for all patients not amenable to attempted resection. If this fails, the $\mathrm{CP}$ technique may be used to place the endoprosthesis, with the advantage over pure percutaneous placement of a smaller bore liver puncture and presumably less bleeding and bile leakage. ${ }^{1315}$

In this series of 190 patients treated endoscopically, the only group with potentially resectable disease was those with type I cholangiocarcinoma $(n=25)$. Eighteen of these patients were over the age of 70 years, four had intrahepatic metastases, and 12 had strong medical contraindications to surgery. The rarity of potentially resectable hilar cancer is well illustrated by Bismuth's series of 213 patients referred to a specialist liver surgical unit over 25 years, ${ }^{3}$ with only $16(7 \cdot 5 \%)$ patients undergoing curative resection.

Overall, the results in this series of patients with proximal malignant biliary obstruction treated by endoscopic endoprosthesis insertion were similar to results of previous series. ${ }^{1820} 26$ Successful stenting was achieved in $170(89 \%)$ patients and successful drainage of the biliary 
tree in $155(82 \%)$. Early complications occurred overall in 36 patients $(19 \%)$, and $41(22 \%)$ died within 30 days. Cholangitis was the principal complication occurring in 13 patients $(7 \%)$. Patients with failed stent insertion or drainage in this series were generally managed further by conservative means only, although three had external drainage. It is probable that the technical success rate of stent placement and the drainage success rate could have been increased if all patients had had the combined procedure performed after initial or second endoscopic failure. However, continuation of palliative attempts was deemed inappropriate for 16 patients because of a very poor clinical state (Table II).

There are two prominent areas of difference between this and previous series. Firstly, the immediate periprocedural cholangitis rate was lower than previously reported. ${ }^{2026}$ This was probably the result of our use of prophylactic antibiotics combined with the policy of attempting to inject contrast medium only into the segment subsequently stented. It is our belief that the filling of all segments to obtain a complete 'diagnostic' cholangiogram should be avoided and only sufficient contrast should be injected to obtain imaging of the duct to be stented. Applying this rule, only five patients required placement of a second endoprosthesis for cholangitis. Secondly, only a single endoprosthesis was placed unless either sepsis developed in undrained segments or resolution of cholestasis was insufficient. The former occurred in five patients as described and the latter in a further three patients. That is, a single endoprosthesis provided palliation in all but eight of the patients successfully treated. Most of these second endoprostheses were placed in the left lobe using the $\mathrm{CP}$ procedure. Thus, routine placement of more than one stent to achieve drainage of all obstructed liver segments as advocated by Deviere $e t a l^{26}$ would seem unnecessary, and if attempted but unsuccessful may be the cause of a raised prevalence of cholangitis. ${ }^{27}$ Moreover, the placement of a nasobiliary drain above the stent to irrigate the liver, as advocated by some groups, would seem more likely to cause sepsis in undrained segments than prevent it. ${ }^{26}$ The least intervention for the desired result is always the best.

Distant or local metastatic cancer causing proximal biliary obstruction may be managed in an identical manner to primary malignancy, except that resection is not suitable. The morbidity and mortality associated with endoprosthesis insertion, however, is considerably higher for these patients and the survival is significantly shorter (Fig 2; Table IV). This is undoubtedly because of the influence of the underlying disease, yet even a short term survival may be considerably improved in terms of quality of life by relief from pruritus or nausea. The poor prognosis in this group does, however, demand careful patient selection for treatment and we would not advocate intervention on the basis of serum biochemistry alone.

In conclusion, most patients with unresectable malignant proximal biliary obstruction may be treated successfully with an acceptably low complication rate by the endoscopic insertion of a single endoprosthesis. Both primary and secondary malignancy may be managed successfully. The prevalence of cholangitis is lower than with more complex interventions. Surgical intervention should probably be limited to the minority of younger and fitter patients in whom the tumour seems to be resectable.

A A Polydorou is supported by the State Scholarships Foundation of Greece and J F Dowsett by the Cancer Research Campaign.

The authors thank Mr M J McMahon and Mr E M Elliot of the Department of Surgery, Leeds General Infirmary, for their help in Department of Surgery, Leeds
preparation of this manuscript.

1 Dowsett JF, Polydorou AA, Vaira D, et al. Endoscopic owst $\mathrm{JF}$, Polydorou $\mathrm{AA}$, Vaira $\mathrm{D}$, et al. Endoscopic A review of 641 consecutive patients. Gut 1988; 29: 1458 .

2 Blumgart LH, Hadjis NS, Benjamin IS, Beazley R. Surgical approaches to cholangiocarcinoma at confluence of hepatic ducts. Lancet 1984; i: 66-70.

3 Bismuth H, Castaing D, Traynor O. Resection or palliation priority of surgery in the treatment of hilar cancer. World $\mathcal{f}$ Surg 1988; 12: 39-47.

4 Bismuth H, Corlette MB. Intrahepatic cholangioenteric anastomosis in carcinoma of the hilus of the liver. Surg Gynecol Obstet 1975; 140: 170-8.

5 Nakayama T, Ikeda A, Okuda K. Percutaneous transhepatic drainage of the biliary tract: techniques and results in 104 cases. Gastroenterology 1978; 74: 554-9.

6 Soehendra N, Reyders-Frederix V. Palliative bile duct drain age - a new endoscopic method of introducing a transpape - a new endoscopic method of introd drain. Endoscopy 1980; 12: 8-11.

7 Soehendra N, Grimm H. Endoscopic retrograde drainage for bile duct cancer. World f Surg 1988; 12: 85-90.

8 Burcharth F, Jensen LI, Olesen K. Endoprosthesis for interna drainage of the biliary tract. Gastroenterology 1979; 77: 133-7.

9 McPherson GAD, Benjamin IS, Hodgson HJF, Bowley NB, Allison DJ, Blumgart LH. Preoperative percutaneous transhepatic biliary drainage: the results of a controlled trial. $\mathrm{BrF}$ Surg 1984; 71: 371-5.

10 Mayer WC, Scott Jones R. Internal radiation for bile duct cancer. World $\mathcal{F}$ Surg 1988; 12: 99-104.

11 Levitt MD, Laurence BH, Cameron F, Klemp PFB. Trans papillary iridium-192 wire in the treatment of malignant bile duct obstruction. Gut 1988; 29: 149-52.

12 Speer AG, Cotton PB, Russell RCG, et al. Randomised trial of endoscopic versus percutaneous stent insertion in malignan obstructive jaundice. Lancet 1987; ii: 57-62.

13 Dowsett J, Vaira D, Hatfield A, et al. Endoscopic biliary therapy using combined percutaneous and endoscopic techniques. Gastroenterology 1989; 96: 1180-6.

14 Cotton PB. Endoscopic method for relief of malignant obstructive jaundice. World F Surg 1984; 8: 854-61.

15 Dowsett JF, Vaira D, Hatfield ARW, et al. Endoscopic biliary therapy using combined percutaneous and endoscopic techniques. Gastroenterology 1989; 96: 1180-6.

16 Laurence BH, Cotton PB. Decompression of malignant biliary obstruction by duodenoscopic intubation of bile ducts. $B M \mathcal{J}$ 1980; 280: 522-3.

17 Cotton PB. Duodenoscopic placement of biliary prostheses to relieve malignant obstructive jaundice. Br $\mathcal{F}$ Surg 1982; 69: 501-3.

18 Leung JWC, Emery R, Cotton PB, Russell RCG, Vallon AG, Mason RR. Management of malignant obstructive jaundice at The Middlesex Hospital. $B r \mathcal{F}$ Surg 1983; 70: 584-6.

19 Pichlmayer R, Ringe B, Lauchart W, Bechstein WO, Gubernatis $G$, Wagner $E$. Radical resection and liver grafting as the two main components of surical strategy in the treas two main components of surgical strategy in the treatment of

proximal bile duct cancer. World f Surg 1988; 12: 68-77
20 Tytgat GNJ, Bartelsman JFWM, Den Hartog Jaker FCA Huibregste K, Mathus-Vliegen EMH. Upper intestinal and Huibregste K, Mathus-Vliegen EMH. Upper intestinal and
biliary tract endoprosthesis. Dig Dis Sci 1986; 31: 57-76.

21 Miyazaki K, Nagafuchi K, Nakayama F. Bypass procedure for bile duct cancer. World f Surg 1988; $12: 64-7$.

22 Black K, Hanna SS, Langer B, Jirch DW, Rider WD Management of carcinoma of the extrahepatic bile ducts. Can F Surg 1978; 21 : 542 .

23 Kiil J, Kruse A, Rokkjaer M. Endoscopic biliary drainage. Brf Surg 1987; 74: 1087-90.

24 Riemann JF, Lux G, Rasch W, Beickert-Sterba A. Non surgical biliary drainage - technique, indications and results. Endoscopy 1981; 13: 157-61.

25 Choi TK, Fan ST, Lai ECS, Wong J. Malignant hilar biliary obstruction treated by segmental bilioenteric anastomosis. obstruction treated by se

26 Deviere J, Baize M, De Toeuf J, Cremer M. Long-term followup of patients with hilar malignant stricture treated by up of patients with hilar malignant stricture treated by scopy 1988; 34: 95-101.

27 Deviere J, Motte S, Dumonceau JM, Serruys E, Thys JP Cremer M. Risk factors for septisaemia after biliary andoprosthesis placement (BEP). Endoscopy 1988; 20: 79 . 